\title{
The normal subgroup structure of ZM-groups
}

\author{
Marius Tărnăuceanu \\ February 17, 2015
}

\begin{abstract}
The main goal of this note is to determine and to count the normal subgroups of a ZM-group. We also indicate some necessary and sufficient conditions such that the normal subgroups of a ZM-group form a chain.
\end{abstract}

MSC (2010): Primary 20D30; Secondary 20D60, 20E99.

Key words: ZM-groups, normal subgroups, chains.

\section{Introduction}

The starting point for our discussion in given by the paper [2, where the class $\mathcal{G}$ of finite groups that can be seen as cyclic extensions of cyclic groups has been considered. The main theorem of [2] furnishes an explicit formula for the number of subgroups of a group contained in $\mathcal{G}$. In particular, this number is computed for several remarkable subclasses of $\mathcal{G}$ : abelian groups of the form $\mathbb{Z}_{m} \times \mathbb{Z}_{n}$, dihedral groups $D_{2 m}$, and Zassenhaus metacyclic groups (ZM-groups, in short).

In group theory the study of the normal subgroups of (finite) groups plays a very important role. So, the following question concerning the class $\mathcal{G}$ is natural:

Which is the number of normal subgroups of a group in $\mathcal{G}$ ?

The purpose of the current note is to answer partially this question, by finding this number for the above three subclasses of $\mathcal{G}$. Since all subgroups of an abelian group are normal, for the first subclass the answer is given by [2]. The 
number of normal subgroups of the dihedral group $D_{2 m}$ is also well-known, namely $\tau(m)+1$ if $m$ is odd, and $\tau(m)+3$ if $m$ is even (as usually, $\tau(m)$ denotes the number of distinct divisors of $m \in \mathbb{N}^{*}$ ). Therefore we will focus only on describing and counting the normal subgroups of ZM-groups.

Most of our notation is standard and will not be repeated here. Basic definitions and results on group theory can be found in [5, 6, 8]. For subgroup lattice theory we refer the reader to [7, 9].

First of all, we recall that a ZM-group is a finite group with all Sylow subgroups cyclic. By [5], such a group is of type

$$
\mathrm{ZM}(m, n, r)=\left\langle a, b \mid a^{m}=b^{n}=1, b^{-1} a b=a^{r}\right\rangle,
$$

where the triple $(m, n, r)$ satisfies the conditions

$$
\operatorname{gcd}(m, n)=\operatorname{gcd}(m, r-1)=1 \quad \text { and } \quad r^{n} \equiv 1(\bmod m) .
$$

It is clear that $|\mathrm{ZM}(m, n, r)|=m n, \mathrm{ZM}(m, n, r)^{\prime}=\langle a\rangle$ (consequently, we have $\left.\left|\mathrm{ZM}(m, n, r)^{\prime}\right|=m\right)$ and $\operatorname{ZM}(m, n, r) / \mathrm{ZM}(m, n, r)^{\prime}$ is cyclic of order $n$. One of the most important (lattice theoretical) property of the ZM-groups is that these groups are exactly the finite groups whose poset of conjugacy classes of subgroups forms a distributive lattice (see Theorem A of [1]). We infer that they are DLN-groups, that is groups with distributive lattice of normal subgroups.

The subgroups of $\mathrm{ZM}(m, n, r)$ have been completely described in [2]. Set

$$
L=\left\{\left(m_{1}, n_{1}, s\right) \in \mathbb{N}^{3}\left|m_{1}\right| m, n_{1}\left|n, s<m_{1}, m_{1}\right| s \frac{r^{n}-1}{r^{n_{1}}-1}\right\} .
$$

Then there is a bijection between $L$ and the subgroup lattice $L(\operatorname{ZM}(m, n, r))$ of $\mathrm{ZM}(m, n, r)$, namely the function that maps a triple $\left(m_{1}, n_{1}, s\right) \in L$ into the subgroup $H_{\left(m_{1}, n_{1}, s\right)}$ defined by

$$
H_{\left(m_{1}, n_{1}, s\right)}=\bigcup_{k=1}^{\frac{n}{n_{1}}} \alpha\left(n_{1}, s\right)^{k}\left\langle a^{m_{1}}\right\rangle=\left\langle a^{m_{1}}, \alpha\left(n_{1}, s\right)\right\rangle,
$$

where $\alpha(x, y)=b^{x} a^{y}$, for all $0 \leq x<n$ and $0 \leq y<m$. Remark also that $\left|H_{\left(m_{1}, n_{1}, s\right)}\right|=\frac{m n}{m_{1} n_{1}}$, for any $s$ satisfying $\left(m_{1}, n_{1}, s\right) \in L$.

By using this result, we are able to describe the normal subgroup structure of $\operatorname{ZM}(m, n, r)$. 
Theorem 1. The normal subgroup lattice $N(\operatorname{ZM}(m, n, r))$ of $\mathrm{ZM}(m, n, r)$ consists of all subgroups

$$
H_{\left(m_{1}, n_{1}, s\right)} \in L(\operatorname{ZM}(m, n, r)) \text { with }\left(m_{1}, n_{1}, s\right) \in L^{\prime},
$$

where

$$
L^{\prime}=\left\{\left(m_{1}, n_{1}, s\right) \in \mathbb{N}^{3}\left|m_{1}\right| \operatorname{gcd}\left(m, r^{n_{1}}-1\right), n_{1} \mid n, s=0\right\} \subseteq L .
$$

We infer that, for every $m_{1} \mid m$ and $n_{1} \mid n, \mathrm{ZM}(m, n, r)$ possesses at most one normal subgroup of order $\frac{m n}{m_{1} n_{1}}$. In this way, all normal subgroups of $\mathrm{ZM}(m, n, r)$ are characteristic. In particular, the above theorem allows us to count them.

Corollary 2. The following equality holds

$$
|N(\mathrm{ZM}(m, n, r))|=\sum_{n_{1} \mid n} \tau\left(\operatorname{gcd}\left(m, r^{n_{1}}-1\right)\right) .
$$

In the following we will denote by $d$ the multiplicative order of $r$ modulo $m$, that is

$$
d=\min \left\{k \in \mathbb{N}^{*} \mid r^{k} \equiv 1(\bmod m)\right\}
$$

Clearly, the sum in the right side of (1) depends on $d$. For $m$ or $n$ primes, this sum can be easily computed.

Corollary 3. If $m$ is a prime, then

$$
|N(\mathrm{ZM}(m, n, r))|=\tau(n)+\tau\left(\frac{n}{d}\right),
$$

while if $n$ is a prime, then

$$
|N(\mathrm{ZM}(m, n, r))|=\tau(m)+1 .
$$

Mention that the number of normal subgroups of the dihedral group $D_{2 m}$ with $m$ odd can be obtained from (3), by taking $n=2$.

Next we will focus on finding the triples $(m, n, r)$ for which $N(\operatorname{ZM}(m, n, r))$ becomes a chain.

Theorem 4. The normal subgroup lattice $N(\mathrm{ZM}(m, n, r))$ of $\mathrm{ZM}(m, n, r)$ is a chain if and only if either $m=1$ and $n$ is a prime power, or both $m$ and $n$ are prime powers and $\operatorname{gcd}\left(m, r^{k}-1\right)=1$ for all $1 \leq k<n$. 
Remark that Theorem 4 gives a method to construct finite (both abelian and nonabelian) groups whose lattices of normal subgroups are chains of prescribed lengths.

Finally, we indicate an open problem with respect to the above results.

Open problem. Describe and count the normal subgroups of an arbitrary finite group contained in $\mathcal{G}$. Also, extend these problems to arbitrary finite metacyclic groups, whose structure is well-known (see, for example, [4]).

\section{Proofs of the main results}

Proof of Theorem 1. First of all, we observe that under the notation in Section 1 we have

$$
\alpha\left(x_{1}, y_{1}\right) \alpha\left(x_{2}, y_{2}\right)=\alpha\left(x_{1}+x_{2}, r^{x_{2}} y_{1}+y_{2}\right)
$$

This implies that

$$
\alpha(x, y)^{k}=b^{k x} a^{y \frac{r^{k x}-1}{r^{x}-1}}, \text { for all } k \in \mathbb{Z} \text {, and } \alpha(x, y)^{-1}=\alpha\left(-x,-r^{-x} y\right) .
$$

Since

$$
\alpha(x, y)^{-1} \alpha\left(n_{1}, s\right) \alpha(x, y)=\alpha\left(n_{1}, t_{x, y}\right), \text { where } t_{x, y}=-r^{n_{1}} y+r^{x} s+y
$$

one obtains

$$
\begin{aligned}
H_{\left(m_{1}, n_{1}, s\right)}^{\alpha(x, y)} & =\alpha(x, y)^{-1} H_{\left(m_{1}, n_{1}, s\right)} \alpha(x, y)= \\
& =\bigcup_{k=1}^{\frac{n}{n_{1}}} \alpha(x, y)^{-1} \alpha\left(n_{1}, s\right)^{k} \alpha(x, y)^{-1}\left\langle a^{m_{1}}\right\rangle= \\
& =\bigcup_{k=1}^{\frac{n}{n_{1}}}\left(\alpha(x, y)^{-1} \alpha\left(n_{1}, s\right) \alpha(x, y)\right)^{k}\left\langle a^{m_{1}}\right\rangle= \\
& =\bigcup_{k=1}^{\frac{n}{n_{1}}} \alpha\left(n_{1}, t_{x, y}\right)^{k}\left\langle a^{m_{1}}\right\rangle=H_{\left(m_{1}, n_{1}, t_{x, y}\right)}
\end{aligned}
$$


with the convention that $t_{x, y}$ is possibly replaced by $t_{x, y} \bmod m_{1}$. Then $H_{\left(m_{1}, n_{1}, s\right)}$ is normal in $\operatorname{ZM}(m, n, r)$ if and only if we have $t_{x, y} \equiv s\left(\bmod m_{1}\right)$, or equivalently

$$
m_{1} \mid s\left(r^{x}-1\right)-y\left(r^{n_{1}}-1\right),
$$

for all $0 \leq x<n$ and $0 \leq y<m$. Take $x=0$ in (4). It follows that $m_{1} \mid y\left(r^{n_{1}}-1\right)$, for all $0 \leq y<m$, and so $m_{1} \mid r^{n_{1}}-1$. We get $m_{1} \mid s\left(r^{x}-1\right)$, for all $0 \leq x<n$. By putting $x=1$ and using the equality $\operatorname{gcd}(m, r-1)=1$, it results $m_{1} \mid s$. But $s<m_{1}$, therefore $s=0$. Hence we have proved that the subgroup $H_{\left(m_{1}, n_{1}, s\right)}$ is normal if and only if $m_{1} \mid \operatorname{gcd}\left(m, r^{n_{1}}-1\right)$ and $s=0$, as desired.

Proof of Theorem 4. Suppose first that $N(\operatorname{ZM}(m, n, r))$ is a chain. Then $\mathrm{ZM}(m, n, r)$ is a monolithic group, that is it possesses a unique minimal normal subgroup. By Theorem 5.9 of [3] it follows that either $m=1$ and $n$ is a prime power, or $m$ is a prime power and $r^{k} \not \equiv 1(\bmod m)$ for all $1 \leq k<n$. On the other hand, we observe that $N(\operatorname{ZM}(m, n, r))$ contains the sublattice

$$
L_{1}=\left\{H_{\left(1, n_{1}, 0\right)}\left|n_{1}\right| n\right\},
$$

which is isomorphic to the lattice of all divisors of $n$. Thus $n$ is a prime power, too. In order to prove the last assertion, let us assume that

$$
\operatorname{gcd}\left(m, r^{k}-1\right)=m_{1} \neq 1
$$

for some $1 \leq k<n$ and consider $k$ to be minimal with this property. It follows that $k \mid n$. Then the subgroup $H_{\left(m_{1}, k, 0\right)}$ belongs to $N(\operatorname{ZM}(m, n, r))$, but it is not comparable to $H_{(1, n, 0)}=\mathrm{ZM}(m, n, r)^{\prime}$, a contradiction.

Conversely, if the triple $(m, n, r)$ satisfies one of the conditions in Theorem 4 , then $N(\operatorname{ZM}(m, n, r))$ is either a chain of length $v$ for $m=1$ and $n=q^{v}(q$ prime), namely

$$
H_{\left(1, q^{v}, 0\right)} \subset H_{\left(1, q^{v-1}, 0\right)} \subset \cdots \subset H_{(1,1,0)},
$$

or a chain of length $u+v$, for $m=p^{u}$ and $n=q^{v}$ ( $p, q$ primes), namely

$$
H_{\left(p^{u}, q^{v}, 0\right)} \subset H_{\left(p^{u-1}, q^{v}, 0\right)} \subset \cdots \subset H_{\left(1, q^{v}, 0\right)} \subset H_{\left(1, q^{v-1}, 0\right)} \subset \cdots \subset H_{(1,1,0)} .
$$

This completes the proof.

Acknowledgements. The author is grateful to the reviewer for its remarks which improve the previous version of the paper. 


\title{
References
}

[1] Brandl, R., Cutolo, G., Rinauro, S., Posets of subgroups of groups and distributivity, Boll. U.M.I. 9-A (1995), 217-223.

[2] Calhoun, W.C., Counting subgroups of some finite groups, Amer. Math. Monthly 94 (1987), 54-59.

[3] De Medts, T., Tărnăuceanu, M., Pseudocomplementation in (normal) subgroup lattices, Comm. Algebra 39 (2011), 247-262, doi: 10.1080/00927870903527493.

[4] Hempel, C.E., Metacyclic groups, Comm. Algebra 28 (2000), 3865-3897.

[5] Huppert, B., Endliche Gruppen, I, Springer Verlag, Berlin, 1967.

[6] Isaacs, I.M., Finite group theory, Amer. Math. Soc., Providence, R.I., 2008.

[7] Schmidt, R., Subgroup lattices of groups, de Gruyter Expositions in Mathematics 14, de Gruyter, Berlin, 1994.

[8] Suzuki, M., Group theory, I, II, Springer Verlag, Berlin, 1982, 1986.

[9] Tărnăuceanu, M., Groups determined by posets of subgroups, Ed. Matrix Rom, Bucureşti, 2006.

\author{
Marius Tărnăuceanu \\ Faculty of Mathematics \\ "Al.I. Cuza" University \\ Iaşi, Romania \\ e-mail: tarnauc@uaic.ro
}

